\title{
A Chronic Post-Surgical Infectious Lumbar Fistula Providing Protection against Imminent Morbidity: A Case Report and Literature Review
}

\author{
Jacob L. Van Orman1*, Paul E. Kaloostian², Mojdeh Najle-Rahim³, Joseph L. Vanderlinden4, \\ Samer S. Ghostine ${ }^{2}$
}

\author{
${ }^{1}$ University of California Riverside School of Medicine, Riverside, CA, USA \\ ${ }^{2}$ Department of Neurosurgery, University of California at Riverside School of Medicine, Riverside, CA, USA \\ ${ }^{3}$ Department of Infectious Disease, Riverside Community Hospital, Riverside, CA, USA \\ ${ }^{4}$ Department of Vascular Surgery, Riverside Community Hospital, Riverside, CA, USA \\ Email: ${ }^{* j v a n o 001 @ m e d s c h . u c r . e d u ~}$
}

How to cite this paper: Van Orman, J.L., Kaloostian, P.E., Najle-Rahim, M., Vanderlinden, J.L. and Ghostine, S.S. (2017) A Chronic Post-Surgical Infectious Lumbar Fistula Providing Protection against Imminent Morbidity: A Case Report and Literature Review. Open Journal of Modern Neurosurgery, 7, 41-48.

https://doi.org/10.4236/ojmn.2017.73005

Received: April 6, 2017

Accepted: June 20, 2017

Published: June 23, 2017

Copyright $\odot 2017$ by authors and Scientific Research Publishing Inc. This work is licensed under the Creative Commons Attribution International License (CC BY 4.0).

http://creativecommons.org/licenses/by/4.0/

\begin{abstract}
Vertebral osteomyelitis (VOM) and spinal epidural abscesses (SEA) are rare infections on the spinal column, more prominently seen in intravenous drug users, diabetics and the immunosuppressed. We report an extremely rare case of a patient with a chronic history of lumbar post-surgical cutaneo-spinal fistulous infection that was unresponsive to conventional antibiotic therapy and ultimately required surgical debridement and spinal re-instrumentation. The novelty of this case was the chronic nature of the patient's condition that was made possible only by a fortunate post-surgical cutaneo-spinal fistula that withdrew infected particles away from the neurological structures and thus prevented imminent compressive and neurological deficits, and possible eventual death. This rare case highlights the need for prompt surgical evaluation and intervention in patients with progressive VOM or SEA with associated cutaneo-spinal fistulas, especially in cases where conventional antibiotic treatments have failed. We highly recommend a low threshold for surgical debridement and hardware removal with re-instrumentation as appropriate before a spinal compressive emergency arises and/or sepsis develops.
\end{abstract}

\section{Keywords}

Spinal Epidural Abscess, Vertebral Osteomyelitis, Cutaneo-Spinal Fistula, Spinal Reconstruction 


\section{Introduction}

Vertebral osteomyelitis (VOM) and spinal epidural abscesses (SEA) are relatively rare infectious conditions that attack the spinal column [1] [2] [3]. Incidence is relatively low, as VOM has been approximated to $0.2-2.0$ cases per 100,000 people annually [1]. Certain risk factors such as intravenous (IV) drug usage, diabetes mellitus (DM) and immunosuppression predispose a patient to the development of these diseases [4] [5]. Methicillin sensitive staphylococcus aureus (MSSA) and methicillin resistant staphylococcus aureus (MRSA) are the most common bacterial agents causing VOM and SEA [4] [6]. VOM and SEA with associated post-surgical cutaneo-spinal fistula are exceedingly rare and no consensus exists on management of such pathology. After diagnosis via careful history, physical examination and magnetic resonance imaging (MRI) with and without contrast of the appropriate area of the spine, a careful consideration of the onset of neurological deficits and local spinal instability directs care towards immediate surgical debridement, decompression and stabilization or a more conservative approach with targeted antibiotics [6]. The majority of cases of VOM and SEA without fistula are effectively managed with antibiotics alone, particularly in the absence of acute neurological deficits or spinal instability [4]. In this unique case, this patient developed an iatrogenic post-surgical osteomyelitis with associated cutaneo-spinal fistula which invaded both the hardware and soft tissues of the lumbar spine that was refractory to antibiotic therapy. The now infectious fistulous connection to the skin provided a protective tunnel for infection to clear the body instead of developing into a spinal abscess with compressive neurological deficits. This unique case highlights the profound need for surgical intervention sooner rather than later for treatment of infectious cutaneospinal fistulas as conservative management is ineffective acutely and may delay patient healing.

\section{Clinical Presentation}

A fifty-year-old Hispanic non-diabetic woman without a history of IV drug use and smoking first noted a persistent lower back pain in 2007 which prompted a hospitalization at a hospital in Las Vegas. While there, she was diagnosed with MRSA vertebral osteomyelitis that required posterior lumbar surgical decompression and debridement. Subsequent unremitting pain unresponsive to oral medication led her to seek treatment at another hospital in Arizona in 2013, where an L3 and L4 anterior corpectomy was performed via a left lateral retroperitoneal approach with anterior placement of a femoral ring allograft for L2-5 fusion. An immediate post-operative infection was noted by purulent drainage from a left flank percutaneous fistula tract likely from the site of the postoperative drain. The tract remained chronically patent, discharging yellowish material from a pinpoint fistula. In 2014, she was treated with 6 weeks of IV antibiotics through a PICC line that temporarily suppressed wound drainage at times but did not eradicate the infection. She sought care at different facilities thereafter, receiving unsuccessful oral antibiotic treatments. In August 2015 she 
was referred to our facility.

At admission she complained of intermittent fevers, sweats and chills and a persistent low back pain. She denied nausea, vomiting, chest pain, headache, $\mathrm{SOB}$, and urinary or bowel incontinence. She continued to complain of leakage from her pinpoint fistulous tract along the initial post-surgical drain site. On physical examination, the patient was ambulating without assistance and her vitals were stable. A small ulcer on the level of the left iliac crest in the middle of prior surgical scar was noted to express yellowish discharge with circumferential mild fluctuance. Laboratory results demonstrated a WBC of $10.2 \mathrm{~K} / \mathrm{mm}^{3}$, CRP of $131 \mathrm{mg} / \mathrm{L}$, ESR of $89 \mathrm{~mm} / \mathrm{hr}$, alkaline phosphatase of $318 \mathrm{U} / \mathrm{L}$, glucose of 126 $\mathrm{mg} / \mathrm{dL}$ and lactic acid of $0.38 \mathrm{mmol} / \mathrm{L}$. Blood cultures were negative for growth and a urine analysis was unremarkable. A culture of the pinpoint flank fistulous discharge was positive for MSSA. CT of the abdomen and pelvis demonstrated anterior pseudoarthrosis at the level of L4-5 with failure of the fusion construct. Anatomic alignment was maintained and the spinal canal was within normal limits. Edema, fluid and air pockets in the left inferior iliopsoas extended to the L5 anterior strut graft, consistent with possible ill-defined abscess or inflammatory phlegmon (see Figure 1 and Figure 2).

Treatment was administered per infectious disease recommendations with appropriate combined antibiotic therapy with vancomycin, rifampin and clindamycin. Despite the antibiotic treatment, patient continued with fever spikes to 102.9 F which prompted a neurosurgical decision to operate. The patient underwent an anterior L2-5 revision of fusion with expandable titanium cage,

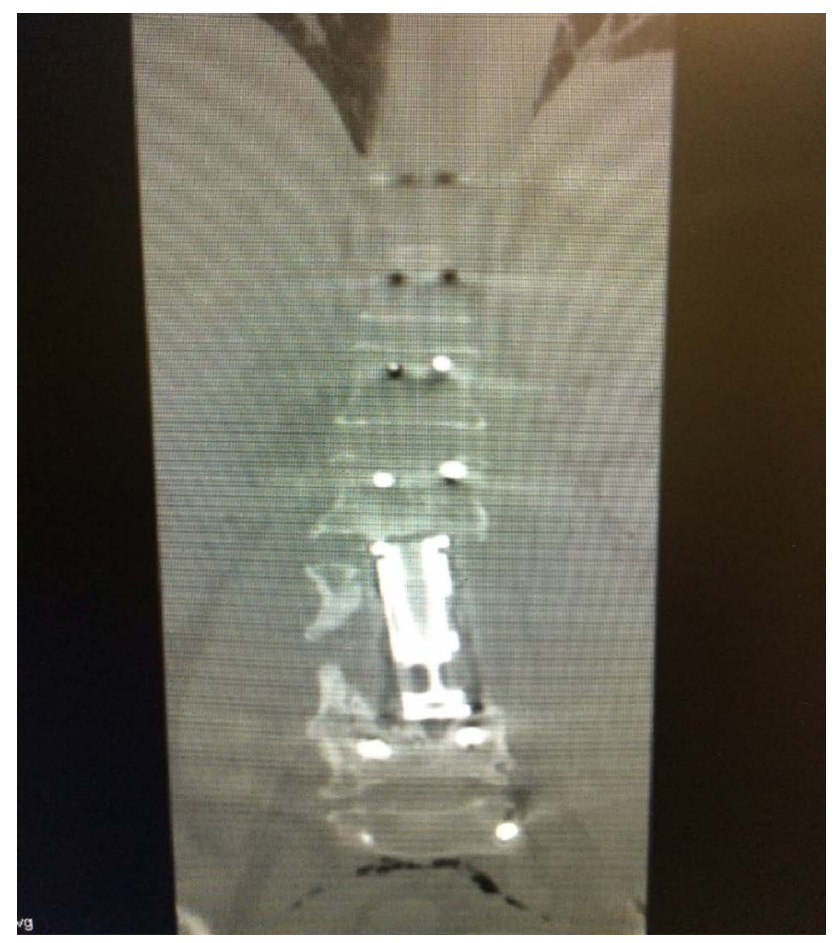

Figure 1. Coronal CT scan demonstrating interbody cage extending over L3 and L4 corpectomy sites with pedicle screw instrumentation above and below. Note the significant pseudoarthrosis with haloing and bony erosion along the L5 superior endplate. 


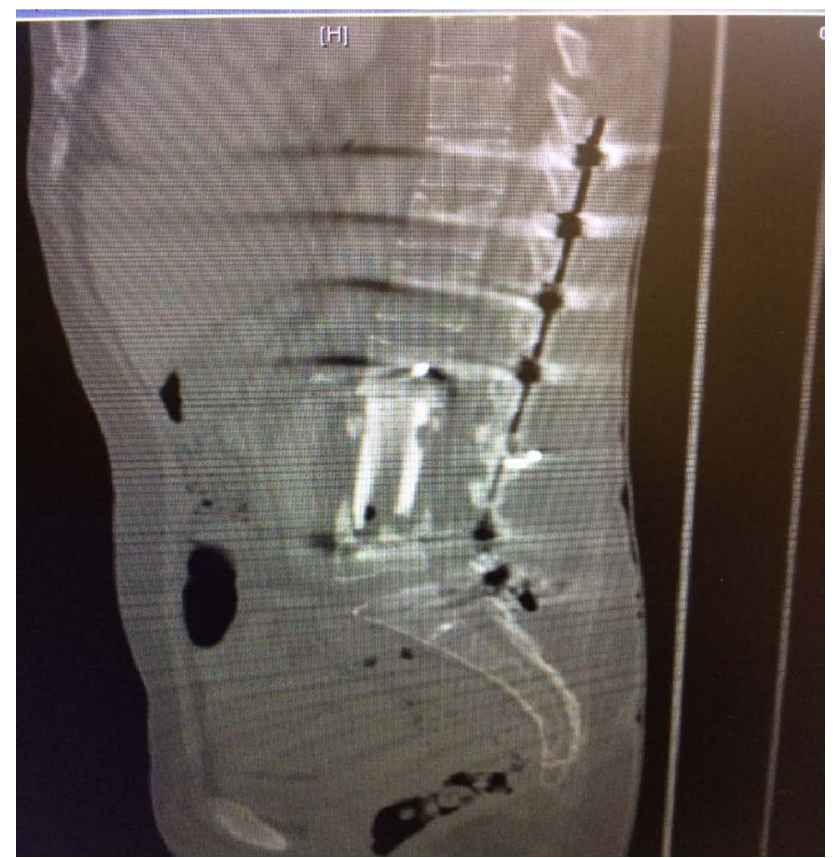

Figure 2. Sagittal CT lumbar spine demonstrating significant pseudoarthrosis due to chronic lingering cutaneo-spinal infectious fistula with osteomyelitis.

removal of prior instrumentation and femoral allograft, and irrigation and drainage of discitis and abscess. An anterior approach was used to access the retroperitoneum where dense adhesions were appreciated as well as very large lymph nodes and purulent drainage. Three specimens were sent for culture. The allograft was exposed, revealing graft fusion at L2-3, and failed fusion at L4-5 with phlegmon present. A window was drilled longitudinally from L2-5, uncovering more purulent material. The phlegmon was dissected free and the femoral ring was dissected into halves, completely removed, and sent for analysis. An expandable titanium cage was fastened tightly to the defect from L2-5. Antibiotic treatment with Ancef and Rifampin was continued post operatively for 6 weeks per infectious disease recommendation and based on cultures from surgery showing MSSA. Patient ESR post operatively decreased to $46 \mathrm{~mm} / \mathrm{hr}$, CRP decreased to $100 \mathrm{mg} / \mathrm{L}$, and WBC decreased to $5.1 \mathrm{~K} / \mathrm{mm}^{3}$. Upon follow up ten months later, patient was neurologically intact without any drainage from her surgical site.

\section{Discussion}

VOM is a condition characterized by an infective process which affects the bony and neurological structures of the spinal column, often with accompanying complications that may include discitis and SEA. Presenting symptoms usually involve back pain, neurological deficits, and fever, with back pain remaining the most common and reliable indication of VOM and SEA [4] [6]. Individuals with a history of IV drug usage, alcohol abuse, low back trauma or surgery, and indwelling vascular devices are at increased risk for these conditions as well as those with a history of diabetes, immunosuppression and renal, hepatic, or cardiac 
comorbidities [4] [5].

Recent analysis of SEA has demonstrated an increasing incidence of these cases in the last 40 years (0.2 - 1.2 cases per 10,000 hospitalizations in the 1970s as compared to 2 to 12.5 cases per 10,000 hospitalization today) [2] [3]. This is probably the consequence of more prevalent IV drug usage, improved diagnostic imaging modalities allowing for earlier disease detection and longer life expectancy in patients with chronic diseases [4] [7]. MSSA and MRSA remain the most common bacterial sources of VOM and SEA [4] [6] [8]. SEA is classified as either a primary or secondary infection. Secondary infections often occur as the consequence of invasive spinal operations or from skin, GU, GI, or oral mucosal primary foci [9]. In cases of secondary abscess, infection travels from the primary foci to the spine hematogenously affecting men more than women and the predominantly attacking thoracolumbar region [4] [6] [10]. A diagnosis is made by consideration of the presenting signs and symptoms, a magnetic resonance imaging (MRI) study, and laboratory work-up including WBC, ESR, CRP, Hgb $\mathrm{A} 1 \mathrm{C}$ and cultures (blood, urine and abscess).

The current literature presents different methods of treating SEA. If the patient experiences acute neurological deficits at $72 \mathrm{hrs}$ or demonstrates an acute spinal deformity, immediate surgical debridement, spinal decompression and stabilization are indicated [4] [6]. If the spinal integrity is intact and neurological deficits are chronic in nature (greater than $72 \mathrm{hrs}$ ), then culturing of the blood and abscess to identify the infective agent should guide antibiotic therapy. Progress is monitored by regular ESR and CRP measurements. Aggressive antibiotic therapy alone is effective in treating a majority of VOM and SEA cases without risk factors for deterioration [5]. An initial blood work-up that demonstrates unstable diabetes mellitus, CRP greater than 115, WBC greater than 12, an age over 65, bacteremia, MRSA infection or ring enhancing lesions on MRI place the patient at higher risk of neurological deterioration and greater likelihood of failing medical therapy [4] [6] [11]. Surgery is indicated in these scenarios. Antibiotic therapies should be culture specific. MRSA infections are typically treated with vancomycin. MSSA infections should be treated with antibiotics that have Gram + staphylococcus coverage which can include Cefazolin and Clindamycin. Rifampin is useful as an adjunct agent for invasive staphylococcal infections. There is no consensus related to the ideal length of treatment [12].

Despite the above mentioned treatments for SEA, post-surgical cutaneo-spinal infectious fistulas are a uniquely different pathology that we hypothesize should be managed differently. There is no clear consensus on how to treat chronic infectious fistulous connections from the epidural space to the skin post-operatively. These are distinctly different than the classical cerebrospinal fluid fistulas that are commonly encountered post surgically with a dural tear. From our unique case, we present a successful approach toward treating this fistulous connection via surgical debridement, removal of all infected foreign material, and use of sterile instrumentation. These fistulas provide a pseudo tunnel or alternate passageway for post-surgical fluid or infection to exit the body instead of 
building up within or around the neural structures. The chronicity of these fistulas may allow for a walled off tunnel that is quite fibrous preventing spread of infection violently to nearby blood vessels and tissues, thus preventing acute neurological and overall medical deterioration. Viola et al. describe 8 patients with whom they identified lingering post-operative infection requiring surgical debridement and removal of hardware with re-instrumentation. No clear protocol for infectious fistula treatment was recommended [13]. Etemadrezaei et al. describe a case of a draining post-surgical lumbar fistula that began 17 years post-surgery. They noted surgical debridement and hardware removal with re-instrumentation. This case notes a chronic underlying infectious fistula over 17 years that presented eventually as an infectious fistulous connection, in contrast to our patient who had continuous drainage (despite antibiotic therapy that would temporarily suppress drainage) over a 3-year period without neurological deficit or sepsis [14].

Spinal stabilization procedures following spinal debridement frequently involved the use of anterior graft materials to complete arthrodesis. The iliac crest bone graft (ICBG) is considered the gold standard for anterior spinal fusion, though complications like pseudoarthrosis can be as high as $48 \%$ in certain fusion procedures [15]. The addition of demineralized bone matrix (DBM) material helps act as a graft extender and enhancer for allografts, though because of its malleable consistency doesn't serve well as a stand-alone graft [15]. The expandable titanium cage offers unique advantages in the correction of spinal defects, namely its resistance to biofilm adherence and reduced rates of post-operative infections as well as easier operative maneuverability and placement because of its expandable properties [16].

\section{Conclusion}

To review, this unique case demonstrates three years of a chronic post-operative cutaneo-spinal fistulous infection originating from the anterior instrumentation of a prior surgery. Systemic symptoms and persistent back pain with drainage were present throughout the case but, remarkably, neurological function and overall health were preserved. We hypothesize this is likely because of the fistula which mitigated the mass effects of the spinal abscess by pulling infected material away from the neural structures and surrounding tissues. Though she did not have acute neurological symptoms, her CRP was elevated at 131 and her condition was not helped with regular antibiotic usage. Surgical debridement and removal of the allograft and replacement with an expandable titanium cage were indicated and implemented to good effect on the patient's condition. Subsequent culture-appropriate antibiotic treatment was initiated with complete healing of the fistulous tract and intact fusion construct months later in follow up. This unique case will help change current management of the chronic lumbar infectious cutaneo-spinal fistulas to a more aggressive surgical approach instead of via conservative treatment, as no definitive paradigm currently exists for such pathology in the literature. We hypothesize that given the chronicity of the fistulous 
tract, conservative management will not be able to close off the fibrous tract and heal the infection. A more invasive surgical approach is needed in these cases to prevent neurological demise and/or death.

\section{References}

[1] Cheung, W.Y. and Luk, K.D. (2012) Pyogenic Spondylitis. International Orthopaedics, 36, 397-404. https://doi.org/10.1007/s00264-011-1384-6

[2] Reihsaus, E., Waldbaur, H. and Seeling, W. (2000) Spinal Epidural Abscess: A MetaAnalysis of 915 Patients. Neurosurgical Review, 23, 175-204.

https://doi.org/10.1007/PL00011954

[3] Darouiche, R.O. (2006) Spinal Epidural Abscess. The New England Journal of Medicine, 355, 2012-2020. https://doi.org/10.1056/NEJMra055111

[4] Ziu, M., Dengler, B., Cordell, D. and Bartanusz, V. (2014) Diagnosis and Management of Primary Pyogenic Spinal Infections in Intravenous Recreational Drug Users. Neurosurgical Focus, 37, E3. https://doi.org/10.3171/2014.6.FOCUS14148

[5] Butler, J.S., Shelly, M.J., Timlin, M., Powderly, W.G. and O’byrne, J.M. (2006) Nontuberculous Pyogenic Spinal Infection in Adults: A 12-Year Experience from a Tertiary Referral Center. Spine, 31, 2695-2700.

https://doi.org/10.1097/01.brs.0000244662.78725.37

[6] Arko, L., Quach, E., Nguyen, V., Chang, D., Sukul, V. and Kim, B.S. (2014) Medical and Surgical Management of Spinal Epidural Abscess: A Systematic Review. Neurosurgical Focus, 37, E4. https://doi.org/10.3171/2014.6.FOCUS14127

[7] Nolla, J.M., Ariza, J., Gómez-Vaquero, C., et al. (2002) Spontaneous Pyogenic Vertebral Osteomyelitis in Nondrug Users. Seminars in Arthritis \& Rheumatism, 31, 271-278. https://doi.org/10.1053/sarh.2002.29492

[8] Shweikeh, F., Saeed, K., Bukavina, L., Zyck, S., Drazin, D. and Steinmetz, M.P. (2014) An Institutional Series and Contemporary Review of Bacterial Spinal Epidural Abscess: Current Status and Future Directions. Neurosurgical Focus, 37, E9. https://doi.org/10.3171/2014.6.FOCUS14146

[9] Zimmerer, S.M., Conen, A., Müller, A.A., et al. (2011) Spinal Epidural Abscess: Aetiology, Predisponent Factors and Clinical Outcomes in a 4-Year Prospective Study. European Spine Journal, 20, 2228-2234. https://doi.org/10.1007/s00586-011-1838-y

[10] Chan, C.T. and Gold, W.L. (1998) Intramedullary Abscess of the Spinal Cord in the Antibiotic Era: Clinical Features, Microbial Etiologies, Trends in Pathogenesis, and Outcomes. Clinical Infectious Diseases, 27, 619-626. https://doi.org/10.1086/514699

[11] Tuchman, A., Pham, M. and Hsieh, P.C. (2014) The Indications and Timing for Operative Management of Spinal Epidural Abscess: Literature Review and Treatment Algorithm. Neurosurgical Focus, 37, E8. https://doi.org/10.3171/2014.6.FOCUS14261

[12] O'daly, B.J., Morris, S.F. and O'rourke, S.K. (2008) Long-Term Functional Outcome in Pyogenic Spinal Infection. Spine, 33, E246-E253. https://doi.org/10.1097/BRS.0b013e31816b8872

[13] Viola, R.W., King, H.A., Adler, S.M. and Wilson, C.B. (1997) Delayed Infection after Elective Spinal Instrumentation and Fusion. A Retrospective Analysis of Eight Cases. Spine, 22, 2444-2450. https://doi.org/10.1097/00007632-199710150-00023

[14] Etemadrezaei, H., Zabihyan, S., Shakeri, A. and Ganjeifar, B. (2015) 17-Year-Delayed Fistula Formation after Elective Spinal Instrumentation: A Case Report. Iranian Red Crescent Medical Journal, 17, e28090.

https://doi.org/10.5812/ircmj.17(5)2015.28090 
[15] Tilkeridis, K., Touzopoulos, P., Ververidis, A., Christodoulou, S., Kazakos, K. and Drosos, G.I. (2014) Use of Demineralized Bone Matrix in Spinal Fusion. World Journal of Orthopedics, 5, 30-37. https://doi.org/10.5312/wjo.v5.i1.30

[16] Robinson, Y., Tschoeke, S.K., Kayser, R., Boehm, H. and Heyde, C.E. (2009) Reconstruction of Large Defects in Vertebral Osteomyelitis with Expandable Titanium Cages. International Orthopaedics, 33, 745-749.

https://doi.org/10.1007/s00264-008-0567-2

Submit or recommend next manuscript to SCIRP and we will provide best service for you:

Accepting pre-submission inquiries through Email, Facebook, LinkedIn, Twitter, etc. A wide selection of journals (inclusive of 9 subjects, more than 200 journals) Providing 24-hour high-quality service User-friendly online submission system Fair and swift peer-review system Efficient typesetting and proofreading procedure Display of the result of downloads and visits, as well as the number of cited articles Maximum dissemination of your research work

Submit your manuscript at: http://papersubmission.scirp.org/

Or contact ojmn@scirp.org 